\title{
PRESSURE EFFECTS IN POLYCYCLIC AROMATIC NITROGENATED \\ HETEROCYCLES (PANHS): DIAGNOSTIC QUALITIES AND COSMOBAROMETRY POTENTIAL
}

Short title: PANHs as a cosmobarometer

Wren Montgomery* and Mark A. Sephton

Impacts and Astromaterials Research Centre, Department of Earth Science and Engineering, Imperial College London SW7 2AZ, United Kingdom

*Corresponding author. Email: w.montgomery@imperial.ac.uk; Tel: +44 (0)20 75945185

Keywords: astrochemistry - infrared: general - interplanetary medium - ISM: lines and bands - methods: analytical - techniques: spectroscopic 


\begin{abstract}
The influence of polycyclic aromatic nitrogen heterocycles (PANHs), which have been suggested as contributors to the interstellar IR emission bands, on interstellar emission features is difficult to constrain because their infrared characteristics are strongly similar to those for polycyclic aromatic hydrocarbons (PAHs). One possible solution is to seek a means of visualising the presence of PANHs that provides information which is distinct from that for PAHs. Although PANHs and PAHs have similar infrared characteristics in many settings, this relationship may not be universally maintained. We have used in-situ high pressure synchrotron-source Fourier transform infrared (FTIR) spectroscopy to determine that the responses of two representative molecules, acridine and anthracene, differ at high pressures ( $>$ ca. $1 \mathrm{GPa})$. Because there are a number of high pressure environments that can be remotely observed by infrared spectroscopy they represent a potential to glimpse the distribution of PANHs across the Cosmos.
\end{abstract}




\section{INTRODUCTION}

Polycyclic aromatic nitrogen heterocycles (PANHs) have been suggested as present in a wide variety of space and planetary settings, including the galactic interstellar medium and extragalactic objects (Bauschlicher, Peeters, \& Allamandola 2009; Bernstein et al. 2005; Charnley et al. 2005), and have been identified in carbonaceous globules in meteorites (Bonnet et al. 2015; Saito \& Kimura 2009). PANHs are also predicted to be major components of the atmosphere and surface of Titan (Imanaka et al. 2004; Kim \& Kaiser 2009; Landera \& Mebel 2010; McCord et al. 2006; Quirico et al. 2008) within the category of material referred to as tholins, which are believed to be present across the outer solar system and responsible for the varying reddish hues of bodies such as Titan, Callisto, Pluto, and Charon with the exact composition of the tholin determining the exact color (Cruikshank et al. 2015; Dalle Ore et al. 2011).

The infrared spectra of PANHs of various sizes and structurally similar parental polycyclic aromatic hydrocarbons (PAHs) have been extensively studied both computationally (Bauschlicher, et al. 2009; Bauschlicher, Peeters, \& Allamandola 2008; Langhoff et al. 1998; Mattioda, Hudgins, \& Allamandola 2005; Mattioda et al. 2008) and experimentally (Bernstein, et al. 2005; Boersma et al. 2010; Tielens 2008), although the latter route is hindered by the difficulty of obtaining PANH standards. The relative position and number of substituted nitrogen atoms has been shown to have a predictable if subtle effect on the infrared spectra proposed by some to account for the $6.2 \mu \mathrm{m}$ interstellar medium (ISM) emission band of the interstellar medium (ISM) (Hudgins, Bauschlicher, \& Allamandola 2005). 
Many of the environments in which PANHs are observed or are postulated to be present are affected by pressure. From the transient shock waves from supernovae to the weak effects of impacts on planetary bodies such as Titan, pressures in organic-rich environments in the Cosmos can span a range from vacuum to $10^{9} \mathrm{GPa}$. Table 1 outlines the range of pressures experienced in cosmological processes, planetary formation events, and within planets. For intraplanetary envronments (e.g., subduction and strike-slip faulting) sample values are given for Earth, but there is a large body of literature on scaling these enviroments to exoplanets (Korenaga 2010; Lenardic \& Crowley 2012; O'Neill \& Lenardic 2007; Unterborn et al. 2014).

It has been observed previously that the infrared spectra of the PAH anthracene and the PANH acridine, despite the obvious differences in atomic structure, molecular, and crystal symmetry, are functionally indistinguishable (Fialkovskaya \& Nefedov 1968; Hudgins, et al. 2005; Langhoff, et al. 1998). Although additional small peaks are observed in the spectra of acridine owing to symmetry splitting in the $\mathrm{C}-\mathrm{C}$ stretching and $\mathrm{C}-\mathrm{H}$ bending regions, these are small and difficult to observe without a high signal to noise source such as synchrotron infrared light (Charnley, et al. 2005; Langhoff, et al. 1998). Comprehensive comparative vibrational assignments have been made of both molecular crystals (Brigodiot \& Lebas 1972; Fialkovskaya \& Nefedov 1968).

One possible means of distinguishing between anthracene and acridine in nature is to monitor their infrared response under conditions not normally experienced in the 
laboratory. Recently the field of organic barometry has been initiated by the discovery of distinct responses of dimethylnaphthalane isomers to temperature and pressure (Montgomery, Watson, \& Sephton 2014). It is possible that similar differences may be observed for the structurally similar but chemically distinct anthracene and acridine molecules thereby providing a means of discrimination in certain astrophysical settings. The presence of nitrogen atoms in aromatic species throughout the cosmos is of major importance because they are far more chemically accessible via reactions involving their aromatic carriers in both condensed and solid phases than the quantitatively important but relatively unreactive nitrogen in $\mathrm{N}_{2}$.

Anthracene has been previously studied under high pressure using FTIR spectroscopy (Adams \& Tan 1981), Raman spectroscopy (Zhao, Baer, \& Chronister 1999), fluoresence spectroscopy (Dreger, Lucas, \& Gupta 2003), and X-ray diffraction (Oehzelt et al. 2003; Oehzelt, Resel, \& Nakayama 2002). The conclusion of this body of work is that anthracene is extremely sensitive to non-hydrostatic stresses and that previously reported phase transitions around $2.4 \mathrm{GPa}$ are the result of non-hydrostatic influences rather than a hydrostatic response. Acridine has not been previously studied under either hydrostatic or non-hydrostatic pressures.

Here we have used in-situ high pressure infrared data, collected using a diamond anvil cell and synchrotron source, to study the effects of pressure and temperature on the structurally similar, chemically distinct organic molecular crystals of acridine and anthracene. This work shows that these materials, indistinguishable by infrared 
spectroscopy at ambient conditions, are readily distinguishable at elevated pressures, including characteristic visible colors (Figure 1a-f) and spectral changes. Further, heated high-pressure experiments on acridine show that heat, frequently inseparable from pressure in astrophysical environments, acts as a fixing agent, rendering these changes permanent. Our data suggest a new method for the remote discrimination of PAH and PANH. The distinct responses of anthracene and acridine also provide a new predictable organic response to pressure that can be added to the currently small but potentially useful list of cosmobarometers.

\section{EXPERIMENTAL}

A description of the apparatus and techniques used can be found elsewhere (Jennings, Montgomery, \& Lerch 2010; Montgomery, et al. 2014) and only the salient features are summarized here. A stainless steel gasket with a $200 \mu \mathrm{m}$ diameter sample chamber and a pre-indented thickness of $30 \mu \mathrm{m}$ depth was placed in the diamond anvil cell. This was loaded with a cesium iodide (CsI) window, a ruby and a piece of crystalline sample. CsI is transparent over the range of wavelengths used, so will not add spectral artifacts to the collected spectra. FTIR spectra were collected using synchrotron light from the Swiss Light Source at intervals of approximately $1 \mathrm{GPa}$ from 0.5 to $9.2 \mathrm{GPa}$. The change in the R1 pressure-dependent florescence line of ruby (Jennings, et al. 2010; Mao, Xu, \& Bell 1986) was used to directly measure pressure. Analysis of spectra was performed by removing background and subsequently using Fityk to fit peaks to a standard Gaussian (Wojdyr 2010). 
Figure 1 shows the structures of acridine along with in-situ microphotographs of acridine and anthracene on compression and decompression in the diamond anvil cell. The color change in anthracene (Figure 1d-f), a subtle reddening, has been previously reported as resulting from non-hydrostatic stresses in the sample (Dreger et al. 2009). The finer grain size of the anthracene sample required a larger polycrystalline volume for adequate spectral response: the polycrystallinity somewhat obscures the color change. The more obvious color change in acridine (Figure 1a-c), from a pale off-white color to a vivid orange-red, has not been previously reported. Acridine is slightly softer than anthracene and the sample flowed under pressure, filling the sample chamber and providing the maximum cross-sectional area for observation of the color change.

For each of 3 experiments at approximately 1.0,3.5, and 6.0 GPa, the sample was first pressurized to the target pressure, then heated at approximately $2.5 \mathrm{~K} / \mathrm{min}$. This rate was chosen as a compromise between $1 \mathrm{~K} / \mathrm{min}$, the standard for determining transitions (Montgomery et al. 2008; Montgomery et al. 2005) and the limited time allocated for synchrotron work. In each case, the sample was heated to the maximum allowable temperature (set by the experimental setup) of $623 \mathrm{~K}$, and then cooled at $2.5 \mathrm{~K} / \mathrm{min}$. Spectra were collected every 25 to $50 \mathrm{~K}$. Pressure was continuously monitored during heating and adjusted to account for mechanical expansion of the DAC with heating. Further details on the error in pressure and temperature measurement are provided in previous work (Jennings, et al. 2010). 


\section{RESULTS \& DISCUSSION}

Acridine and anthracene have previously been observed to be practically indistinguishable at ambient conditions using infrared spectroscopy (Fialkovskaya \& Nefedov 1968; Hudgins, et al. 2005; Langhoff, et al. 1998). Vibrational assignments have been made for both molecules and are summarized in Table 2 (Brigodiot \& Lebas 1972). As might be expected for two similarly structured molecules, the assignments overlap, except for the previously mentioned weak vibrations associated with the N-substitution in acridine. Owing to the use of synchrotron-source infrared with a high signal-noise ratio, these weak acridine vibrations are observable in our data, but would not be detectable using a laboratory source or during observational infrared spectroscopy. The spectroscopic difficulties of identifying PANHs in the ISM have been summarized in

other work (Boersma, Bregman, \& Allamandola 2013; Charnley, et al. 2005). Changes to band positions indicate differences in atomic positions, often relating to the breaking of molecular symmetry.

Changes in infrared spectra brought about by N-substitution reactions that produce PANH from PAH have been modelled and measured where samples are readily available. Nsubstitution effects are seen in the $6.2 \mu \mathrm{m}$ peaks, which correspond to the dominant $\mathrm{C}-\mathrm{C}$ stretching mode. The shift of the $6.2 \mu \mathrm{m}$ peak to lower wavelengths is hypothesized to reflect $\mathrm{N}$-substitution inside the PAH structure as opposed to on the outside edge. Multiple N-substitutions are predicted to have a similar peak-broadening effect (Hudgins, et al. 2005; Langhoff, et al. 1998). Although the consquences of varying levels of N- 
substitution have been studied, the effects of pressure on PANHs have not previously been investigated.

Figure 1 shows that, with the application of pressure, acridine and anthracene respond differently. Figure 2 reveals that infrared characteristics of acridine and anthracene, particularly in the mid infrared region of 6-17 $\mu \mathrm{m}\left(600-1650 \mathrm{~cm}^{-1}\right)$, also change in distinct fashion in response to pressure. Individual peaks of interest are numbered throughout. Figure 3 summarizes the central position of each peak as it changes with pressure; consideration of Figures 2 and 3 allows recognition of where new peaks are being formed. Notable examples of new peaks forming can be seen at $16.2 \mu \mathrm{m}$ and13.4 $\mu \mathrm{m}$ in acridine and $13.4 \mu \mathrm{m}$ and $7.6 \mu \mathrm{m}$ in anthracene. For clarity, peaks are referred to by their ambient pressure central peak (or that of the peak from which they have split).

Significant peaks are numbered in Figures 2 and 3. Changes fall into three categories: they occur only in anthracene (3), they occur in both acridine and anthracene $(8,9)$, or they occur only in acridine $(1,2,4,5,6,7,10)$. Very limited and reversible changes in anthracene are associated with the breathing modes of the central ring at $7.6 \mu \mathrm{m}(3)$ and $13.4 \mu \mathrm{m}$ (9), compatible with previous reports of phase transitions in anthracene in a nonhydrostatic environment (Zhao, et al. 1999).

In acridine, the in-plane and out-of-plane $\mathrm{CH}$ vibrations are strongly affected by pressure at $6.8 \mu \mathrm{m}(1), 9.0 \mu \mathrm{m}(4), 10.4 \mu \mathrm{m}(5)$, and $11.6 \mu \mathrm{m}$ (7). These changes suggest that the acridine molecules are collapsing more rapidly with the reduced free volume available as 
the structure is compressed. The rapid decrease in value for the wavelength of vibration (6), which corresponds to a flapping motion of the two "outside" benzene rings further suggests that acridine molecules buckle across the central $\mathrm{C}-\mathrm{N}$ axis when pressure is applied. Also affected by pressure are vibrations in acridine relating to the $\mathrm{N}$-containing ring at $10.9 \mu \mathrm{m}(6), 13.4 \mu \mathrm{m}(8)$, and $16.2 \mu \mathrm{m}(10)$, indicating that the N-substitution creates a weaker ring structure.

For both organic structures, at high pressures all bands shift to lower wavelengths reflecting an increase in energy from compression of nuclei and electrons. Without the application of heat, all pressure-induced transformations were found to be reversible on decompression with only slight hysteresis. Transformations in acridine induced by a combination of pressure and temperature were found to be irreversible at the lower end of the pressures studied (Figure 4). Previous work has previously shown that irreversible changes in anthracene occur outside the pressures and temperatures accessible in our experimental apparatus (8 GPa/650-1500 ${ }^{\circ} \mathrm{C}$ ) (Davydov et al. 2004; Davydov et al. 2006).

At $1.2 \mathrm{GPa}$, heating acridine to $623 \mathrm{~K}$ irreversibly formed new peaks around the $11 \mu \mathrm{m}$ doublet and in the 6-8 $\mu \mathrm{m}$ region where relative peak heights were also affected. At 3.5 $\mathrm{GPa}$, changes for acridine again occurred in the $11 \mu \mathrm{m}$ region, along with substantial changes in the 13-14 $\mu$ m peaks. However, when acridine was heated to $623 \mathrm{~K}$ at $6.0 \mathrm{GPa}$, the changes induced were neither substantial nor irreversible (Figure 4).

Our data suggest that pressure-induced changes in acridine samples that were heated to 
temperatures between 600 and $700 \mathrm{~K}$ can be detected with infrared spectroscopy in the 5 $\mu \mathrm{m}$ to $15 \mu \mathrm{m}$ region. Inspection of Figure 3 shows that at $9.3 \mathrm{GPa}$, the highest pressure measured in these experiments, the new peaks in acridine are moved to the regions 6.6 $\mu \mathrm{m}(1), 7.0 \mu \mathrm{m}$ (2), 8.8-8.9 $\mu \mathrm{m}$ (4), 10.2-10.3 $\mu \mathrm{m}(5-6), 11.2-11.4 \mu \mathrm{m}$ (7), 12.7-13.0 $\mu \mathrm{m}$ $\backslash(8)$, and 15.8-16.0 (10) $\mu \mathrm{m}$. These wavelengths are those at which the new peaks would be observed in post-pressurization environments. Of these peaks, (4), (7), (8), and (10) have the largest relative amplitudes within their spectra. These could contribute to the major emission peaks of the ISM at 8.6, $\mu \mathrm{m} 11.2 \mu \mathrm{m}, 12.7 \mu \mathrm{m}$, and $16.4 \mu \mathrm{m}$ (Tielens 2008). Additionally, broad emission plateaus at $6 \mu \mathrm{m}$ to $9 \mu \mathrm{m}, 11 \mu \mathrm{m}$ to $14 \mu \mathrm{m}$, and 15 $\mu \mathrm{m}$ to $19 \mu \mathrm{m}$ are recognized as part of the ISM (Tielens 2008), and the minor peaks produced under pressure could contribute to these features while explaining the known variation between astronomical regions (van Diedenhoven et al. 2004).

\section{CONCLUSIONS}

The infrared spectra of acridine has characteristic responses to pressure, especially in the regions near the $6.8 \mu \mathrm{m}, 9.0 \mu \mathrm{m}, 10.4 \mu \mathrm{m}, 10.9 \mu \mathrm{m}, 11.6 \mu \mathrm{m}, 13.4 \mu \mathrm{m}$, and $16.2 \mu \mathrm{m}$ peaks, which are associated with $\mathrm{CH}$ and central ring vibrations. PANH such as acridine are potential contributors to the interstellar infrared emission bands but their recognition is hampered by spectral similarities to PAH. The diagnostic infrared responses for acridine at high pressures may provide a solution to the problem of detecting the presence of this type of unit. When heat is added to the pressurized experiments acridine shows irreversible changes at the listed wavelengths that are easily distinguished from the 
structurally similar organic compound anthracene, suggesting that a comparison between acridine and anthracene within IDPs, carbonaceous meteorites and returned extraterrestrial samples may form a new cosmobarometer for astrophysical environments. A new PANH-based cosmobarometer would be a useful addition because these structures appear to be present in a wide variety of astronomical environments, in which high pressures are expected to have played a historical role in the form of shock, impacts or tectonism.

\section{ACKNOWLEDGEMENTS}

This work was supported by STFC Grant ST/K000551/1. The authors are grateful to the

Swiss Light Source, Switzerland for synchrotron access, and especially to Philippe Lerch, beamline scientist at X01DC. We also wish to thank Carole Garnier for very helpful translation of scientific French.

\section{REFERENCES}

Adams, D. M., \& Tan, T.-K. 1981, FaTr, 77, 1711

Allmann, B. P., \& Shearer, P. M. 2009, JGRB, 114, n/a

Anderson, D. 2007, New Theory of the Earth (Cambridge: Cambridge University Press)

Bauschlicher, C. W., Jr, Peeters, E., \& Allamandola, L. J. 2009, ApJ, 697, 311

Bauschlicher, J., C. W. , Peeters, E., \& Allamandola, L. J. 2008, ApJ, 678, 11

Bernstein, M. P., Mattioda, A. L., Sandford, S. A., \& Hudgins, D. M. 2005, ApJ, 626, 909

Boersma, C., Bauschlicher, C. W., Allamandola, L. J., Ricca, A., Peeters, E., \& Tielens, A. G. G. M. 2010, A\&A, 511

Boersma, C., Bregman, J. D., \& Allamandola, L. J. 2013, ApJ, 769, 117

Boersma, C., et al. 2014, ApJS, 211, 8

Bonnet, J.-Y., et al. 2015, Icar, 250, 53

Brigodiot, M., \& Lebas, J. M. 1972, JCPPC, 69, 984

Burrows, A., \& Liebert, J. 1993, RvMP, 65, 301

Chambers, J. E. 2013, Icar, 224, 43

Charnley, S. B., et al. 2005, AdSpR, 36, 137

Cruikshank, D. P., et al. 2015, Icar, 246, 82

Dalle Ore, C. M., et al. 2011, A\&A, 533, A98 
Davison, T. M., Ciesla, F. J., Collins, G. S., \& Elbeshausen, D. 2014, M\&PS, 49, 2252

Davydov, V. A., Rakhmanina, A. V., Agafonov, V., Narymbetov, B., Boudou, J. P., \&

Szwarc, H. 2004, Carbon, 42, 261

Davydov, V. A., Rakhmanina, A. V., Boudou, J. P., Thorel, A., Allouchi, H., \& Agafonov, V. 2006, Carbon, 44, 2015

Dreger, Z. A., Balasubramaniam, E., Gupta, Y. M., \& Joly, A. G. 2009, JPCA, 113, 1489

Dreger, Z. A., Lucas, H., \& Gupta, Y. M. 2003, JPCB, 107, 9268

Fialkovskaya, O. V., \& Nefedov, A. V. 1968, OptSp, 25, 428

Hudgins, D. M., Charles W. Bauschlicher, J., \& Allamandola, L. J. 2005, ApJ, 632, 316

Imanaka, H., et al. 2004, Icar, 168, 344

Jennings, E., Montgomery, W., \& Lerch, P. 2010, JPCB, 114, 15753

Kim, Y. S., \& Kaiser, R. I. 2009, ApJS, 181, 543

Korenaga, J. 2010, ApJL, 725, L43

Landera, A., \& Mebel, A. M. 2010, Faraday Discussions, 147, 479

Langhoff, S. R., Bauschlicher, C. W., Hudgins, D. M., Sandford, S. A., \& Allamandola, L. J. 1998, JPCA, 102, 1632

Lenardic, A., \& Crowley, J. W. 2012, ApJ, 755, 132

Mao, H. K., Xu, J., \& Bell, P. M. 1986, JGRB, 91, 4673

Mattioda, A. L., Hudgins, D. M., \& Allamandola, L. J. 2005, ApJ, 629, 1188

Mattioda, A. L., Rutter, L., Parkhill, J., Head-Gordon, M., Lee, T. J., \& Allamandola, L. J. 2008, ApJ, 680, 1243

McCord, T. B., et al. 2006, P\&SS, 54, 1524

Melosh, H. J. 1989, Impact cratering: a geologic process, Vol. 11 (Tuscon, AZ: Oxford University Press)

Montgomery, W., Crowhurst, J. C., Zaug, J. M., \& Jeanloz, R. 2008, JPCB, 112, 2644

Montgomery, W., Watson, J. S., \& Sephton, M. A. 2014, ApJ, 784, 98

Montgomery, W., Zaug, J. M., Howard, W. M., Goncharov, A. F., Crowhurst, J. C., \&

Jeanloz, R. 2005, JPCB, 109, 19443

O'Neill, C., \& Lenardic, A. 2007, GeoRL, 34, n/a

Oehzelt, M., et al. 2003, JChPh, 119, 1078

Oehzelt, M., Resel, R., \& Nakayama, A. 2002, PhRvB, 66, 174104

Pierazzo, E., \& Chyba, C. F. 1999, M\&PS, 34, 909

Pierazzo, E., \& Melosh, H. J. 2000, M\&PS, 35, 117

Quirico, E., et al. 2008, Icar, 198, 218

Remington, B. A., et al. 1997, PhPl, 4, 1994

Saito, M., \& Kimura, Y. 2009, ApJL, 703, L147

Tielens, A. G. G. M. 2008, ARA\&A, 46, 289

Unterborn, C. T., Kabbes, J. E., Pigott, J. S., Reaman, D. M., \& Panero, W. R. 2014, ApJ, 793, 124

van Diedenhoven, B., Peeters, E., Van Kerckhoven, C., Hony, S., Hudgins, D. M.,

Allamandola, L. J., \& Tielens, A. G. G. M. 2004, ApJ, 61111

Wojdyr, M. 2010, JApCr, 43, 1126

Zang, A., \& Stephansson, O. 2010, Stress Field of the Earth's Crust (Springer)

Zhao, L., Baer, B. J., \& Chronister, E. L. 1999, JPCA, 103, 1728 
Table 1: High pressure environments in the Cosmos

\begin{tabular}{|c|c|c|}
\hline Environment & $\begin{array}{c}\text { Pressure Range, } \\
\text { GPa }\end{array}$ & Reference \\
\hline \multicolumn{3}{|c|}{ Planetary Formation \& Interaction } \\
\hline supernovae & $10^{3}-10^{6}$ & 1,2 \\
\hline $\begin{array}{l}\text { impacts and near } \\
\text { misses }\end{array}$ & $0-10^{4} \mathrm{GPa}$ & 3-7 \\
\hline \multicolumn{3}{|c|}{ Planetary Tectonics } \\
\hline $\begin{array}{l}\text { burial/geotherm } \\
\text { (Earth) }\end{array}$ & $0-1 \mathrm{GPa}$ & 8 \\
\hline $\begin{array}{l}\text { subduction (Earth) } \\
\text { strike-slin faulting }\end{array}$ & $0-136 \mathrm{GPa}$ & 9 \\
\hline $\begin{array}{c}(\Delta \sigma) \\
(\text { Earth })\end{array}$ & $0-1 \mathrm{GPa}$ & 10 \\
\hline
\end{tabular}

References. (1) Burrows \& Liebert 1993; (2) Remington et al. 1997; (3) Chambers 2013; (4) Davison et al. 2014; (5) Melosh 1989; (6) Pierazzo \& Chyba 1999; (7) Pierazzo \& Melosh 2000; (8) Zang \& Stephansson 2010; (9) Anderson 2007; (10)

Allmann \& Shearer 2009 
Table 2: Vibrational assignments of acridine and anthracene at ambient pressure, with comments on high pressure behavior. Vibrations may be visualized in the PAHdb (Boersma et al. 2014). Lines in bold refer to peaks explicitly discussed in the text, reference numbers to the peaks labelled in Figure 2 are given in parentheses.

Abbreviations: $a n=$ anthracene, $a c=$ acridine, $i p=$ in-plane, oop $=$ out-of-plane, $s t r=$ stretching, $\mathrm{vw}=$ very weak, $\mathrm{m}=$ medium, $\mathrm{s}=$ strong, $\mathrm{vs}=$ =very strong, $\mathrm{sh}=$ shoulder.

\begin{tabular}{|c|c|c|c|c|}
\hline $\begin{array}{l}\text { Acridine } \\
\text { peak, } \mu \mathrm{m}\end{array}$ & $\begin{array}{c}\text { Anthracene } \\
\text { peak, } \mu \mathrm{m}\end{array}$ & vibrational assignment & $\begin{array}{c}\text { (Brigodiot } \\
\text { \& Lebas } \\
\text { 1972) } \\
\end{array}$ & $\begin{array}{l}\text { high pressure } \\
\text { behavior }\end{array}$ \\
\hline $6.16 \mathrm{vs}$ & $6.17 \mathrm{~s}$ & ring stretch & $8 b$ & -- \\
\hline $6.35 \mathrm{~m}$ & $6.52 \mathbf{w}$ & ring stretch & $8 \mathrm{a}$ & -- \\
\hline $6.43 \mathrm{~s}$ & -- & ip CH str & $8 \mathrm{a}$ & -- \\
\hline $6.60 \mathrm{~s}$ & -- & ip $\mathrm{CH}$, ring str & $19 \mathrm{a}$ & -- \\
\hline $6.77 w$ & -- & ip CH, ring str & 19b & $\begin{array}{c}\text { ac split (1) }>1 \\
\mathbf{G P a}\end{array}$ \\
\hline $6.84 \mathrm{~s}$ & $6.94 s$ & ip $\mathrm{CH}$, ring str & $19 \mathrm{a}$ & -- \\
\hline $6.94 m$ & -- & ip $\mathrm{CH}$, ring str & $19 b$ & -- \\
\hline $7.14 \mathrm{~s}$ & $7.15 \mathrm{vw}$ & ring breathing & 14 & $\begin{array}{c}\text { ac split }(2)> \\
\text { 3GPa }\end{array}$ \\
\hline $7.28 \mathrm{~m}$ & -- & central ring breathing & e & -- \\
\hline $7.34 w$ & -- & central ring breathing & $\mathrm{e}$ & -- \\
\hline $7.61 \mathrm{~m}$ & $7.61 \mathrm{~s}$ & central ring breathing & e' & $\begin{array}{c}\text { an new peak (3) } \\
>3 \mathrm{GPa}\end{array}$ \\
\hline $7.83 w$ & $7.86 \mathbf{m}$ & ip $\mathrm{CH}$ wag & 3 & -- \\
\hline $7.89 \mathrm{~m}$ & -- & central ring breathing & $\mathrm{e}$ & -- \\
\hline $8.05 \mathrm{vw}$ & -- & ip $\mathrm{CH}$ wag & 3 & -- \\
\hline $8.52 \mathrm{~m}$ & -- & ip CH wag & $9 b$ & -- \\
\hline $8.61 \mathrm{~m}$ & -- & ip ring str & 15 & -- \\
\hline $8.76 \mathrm{~s}$ & $8.73 \mathrm{~m}$ & ip CH wag & $9 b$ & -- \\
\hline $8.89 \mathrm{~m}$ & $8.57 \mathbf{w}$ & ip ring str & 15 & -- \\
\hline $9.01 \mathrm{~m}$ & -- & CH bend & $\delta \mathrm{C}-\mathrm{H}$ & $\begin{array}{c}\text { ac split }(4)> \\
3 \mathrm{GPa}\end{array}$ \\
\hline 9.99s & $10.02 w$ & ip CH str/wag & $18 b$ & -- \\
\hline $10.13 m$ & $10.22 \mathbf{w}$ & oop CH bend & 5 & -- \\
\hline $10.43 \mathrm{vs}$ & $10.46 \mathrm{~s}$ & oop CH bend & $17 b$ & $\begin{array}{c}\text { ac split }(5,6)>1 \\
\text { GPa }\end{array}$ \\
\hline $10.92 \mathrm{~s}$ & -- & ring flap & $\mathrm{d}^{\prime}$ & -- \\
\hline-- & $11.36 \mathrm{vs}$ & oop $\mathrm{CH}$ bend & $\gamma \mathrm{C}-\mathrm{H}$ & -- \\
\hline $11.62 \mathrm{~s}$ & $11.64 w$ & oop CH bend & $10 b$ & $\begin{array}{c}\text { ac split (7) }>5 \\
\mathbf{G P a}\end{array}$ \\
\hline $12.28 \mathrm{~m}$ & $12.36 \mathbf{v w}$ & center ring breathe & $\mathrm{d}$ & -- \\
\hline $12.62 \mathrm{~s}$ & -- & oop CH bend & 4 & -- \\
\hline $13.05 \mathrm{~m}$ & -- & oop $\mathrm{CH}$ bend & 4 & -- \\
\hline $13.4 \mathrm{vs}$ & $13.44 \mathrm{sh}$ & center ring breathe & d & $\begin{array}{c}\text { ac, an split }(8,9) \\
>3 \mathrm{GPa}\end{array}$ \\
\hline $13.59 \mathrm{vs}$ & $13.77 \mathrm{vs}$ & oop $\mathrm{CH}$ bend (umbrella) & 11 & -- \\
\hline $15.30 \mathrm{~m}$ & $15.29 \mathbf{w}$ & center ring breathing & $\mathrm{e}^{\prime}$ & -- \\
\hline $16.20 \mathrm{~m}$ & -- & $(\mathrm{C}-\mathrm{C}) \mathrm{N}$ bend & $\delta(C-C) N$ & $\begin{array}{c}\text { ac split (10) }>3 \\
\text { GPa }\end{array}$ \\
\hline
\end{tabular}




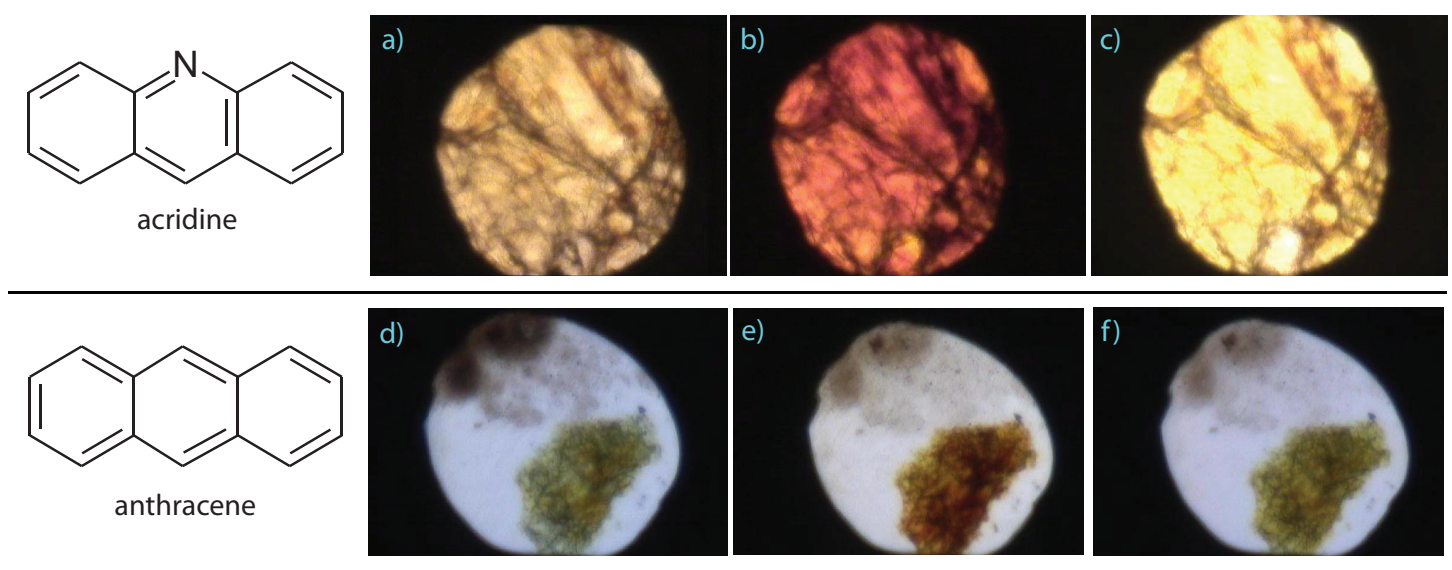

Figure 1: Structure of acridine (top left) and anthracene (bottom left) with microphotographs of in-situ samples: a) acridine at $1.1 \mathrm{GPa}$ b) $9.1 \mathrm{GPa}$ c) $3.5 \mathrm{GPa}$ (on decompression) d) anthracene $2.8 \mathrm{GPa} \mathrm{e}$ ) $7.3 \mathrm{GPa}$ f) $3.1 \mathrm{GPa}$ (on decompression). 


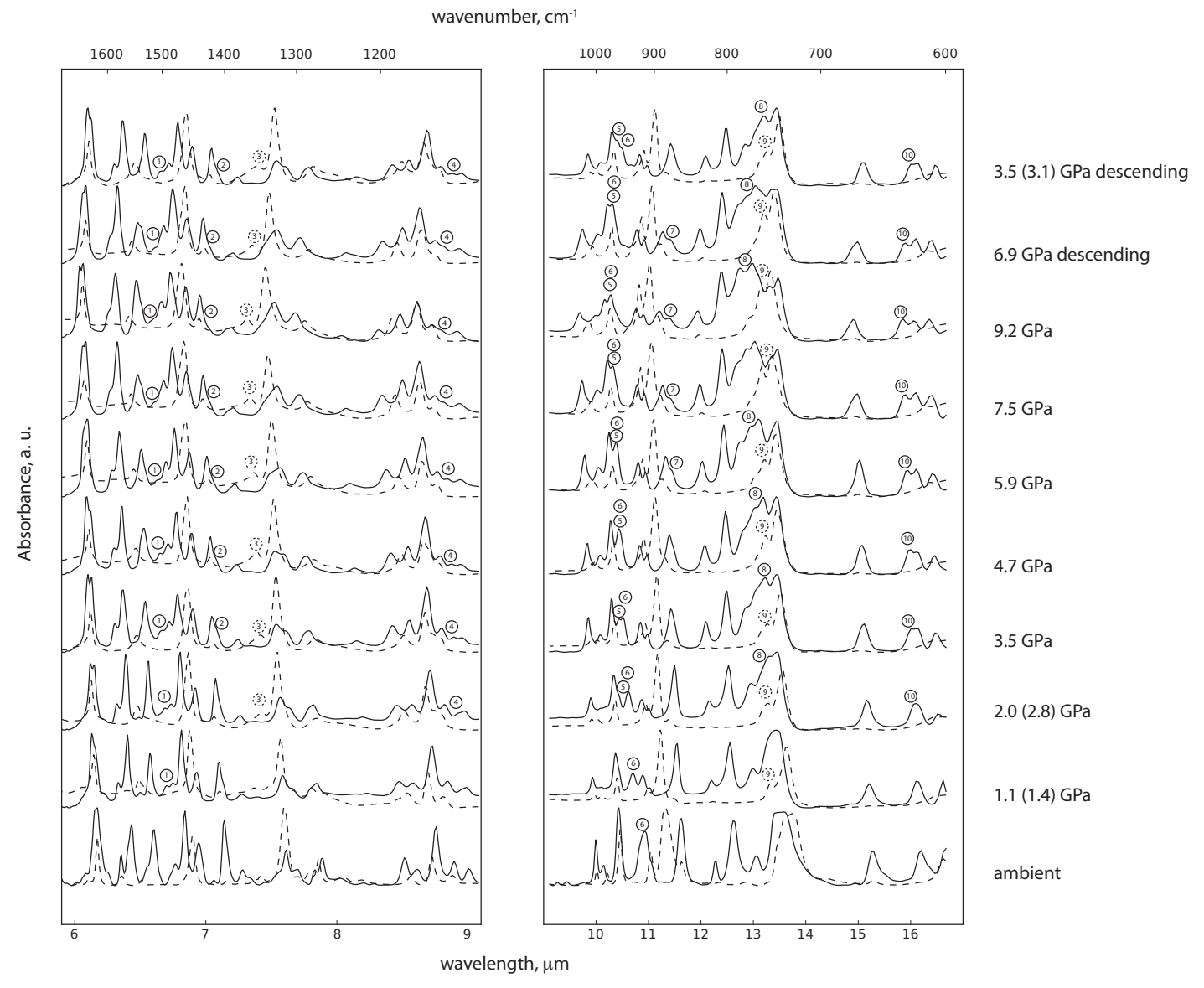

Figure 2: Spectral data for acridine (solid) and anthracene (dashed) for the regions 6-9 $\mu \mathrm{m}\left(1100-1700 \mathrm{~cm}^{-1}\right)$ and 9-17 $\mu \mathrm{m}\left(600-1100 \mathrm{~cm}^{-1}\right)$. Pressures in parentheses refer to anthracene experiments. Numbered peak assignments are given in the text. 

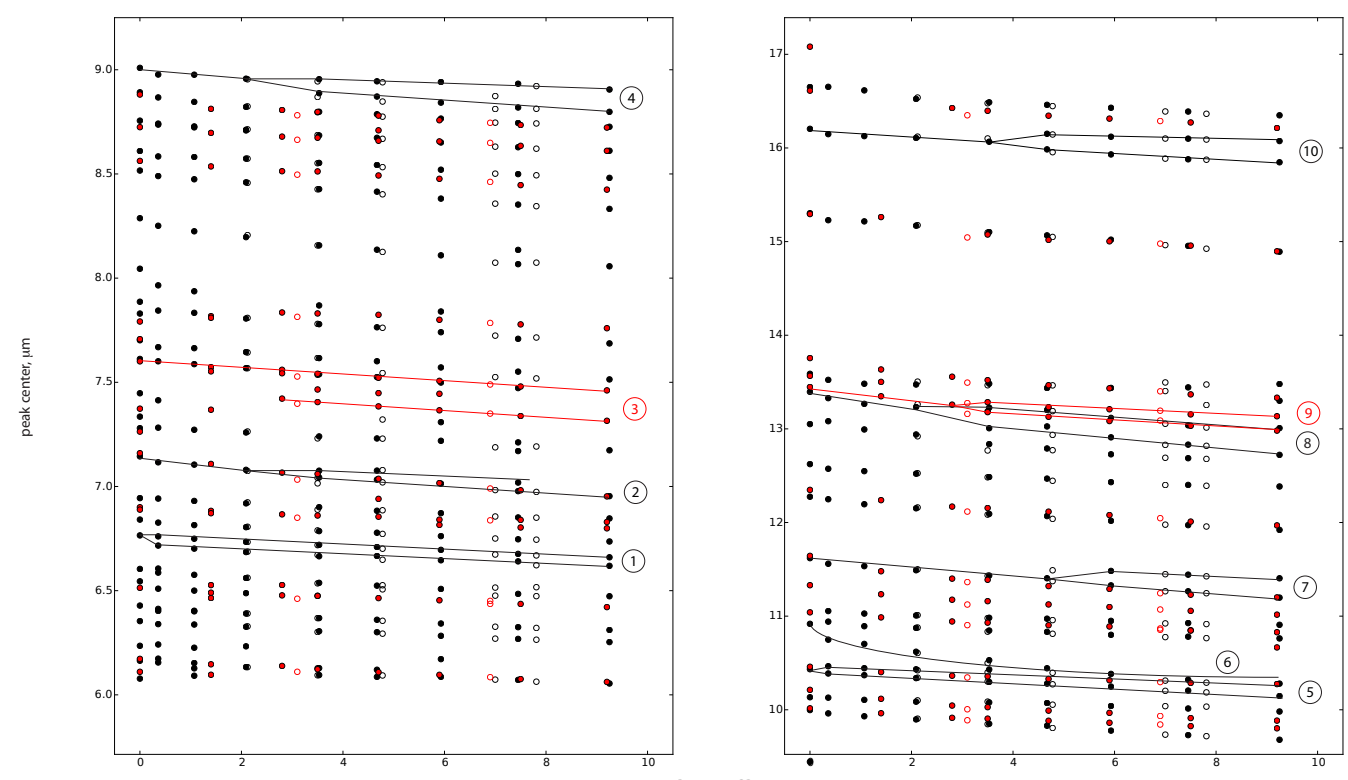

Figure 3: Compression (filled) and decompression (empty) peak fitting data for acridine (black) and anthracene (red). Each data point represents the center of a peak associated with that material at a single pressure. Lines have been drawn to illustrate trends of interest and peak vibrations are assigned to numbers in the text. 

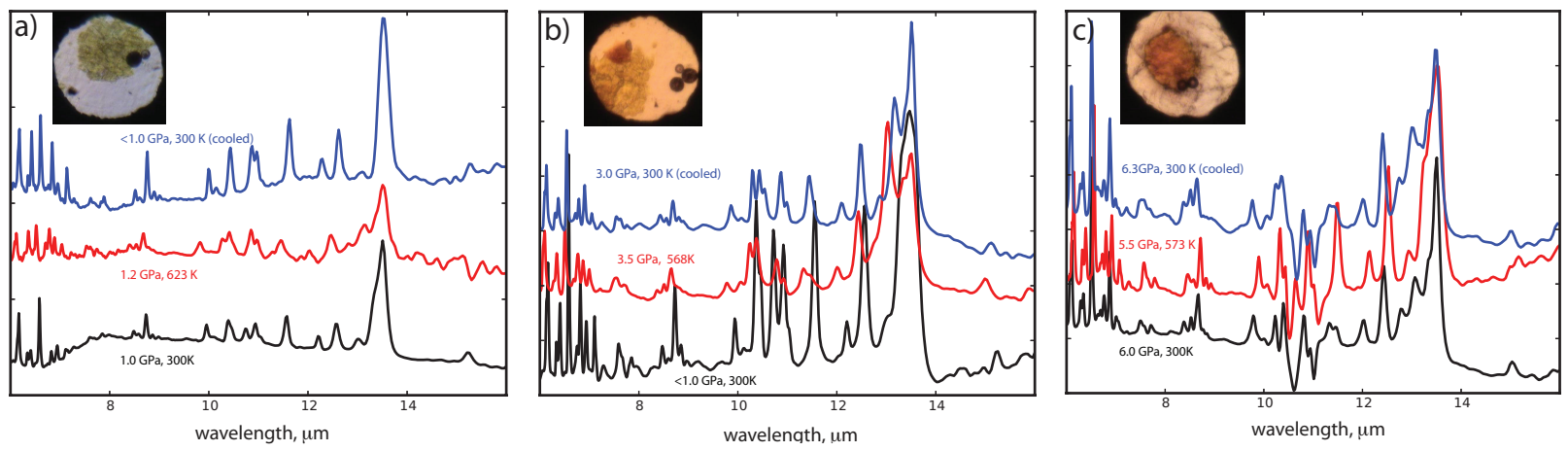

Figure 4: Spectra data for acridine heated to around $623 \mathrm{~K}$ at pressures of a) $1.2 \mathrm{GPa}, \mathrm{b}$ ) 3.5 GPa, and c) $6.0 \mathrm{GPa}$. Inset are microphotographs of the sample at peak temperature for each pressure. Black spectra are the initial sample, red are at peak temperature and blue are on cooling to room temperature. Irreversible changes on decompression are visible in a) at around 7, 10.5, and $11.5 \mu \mathrm{m}$. 